\title{
Uso da prótese vertical expansível de titânio para costela no tratamento da cifose congênita em portadores de mielomeningocele torácica
}

Use of vertical expandable prosthetic of titanium for the rib for treating congenital kyphosis in thoracic meningomyelocele patients

Uso de la prótesis vertical expansible de titanio para costilla en el tratamiento de la cifosis congénita en portadores de mielomeningocele torácico

\author{
Guilherme Rebechi Zuiani' \\ Paulo Tadeu Maia Cavali² \\ Marcus Alexandre Mello Santos ${ }^{3}$ \\ Alexander Junqueira Rossato ${ }^{4}$ \\ Maurício Antonelli Lehoczki ${ }^{5}$ \\ Marcelo Ítalo Risso Neto ${ }^{6}$ \\ Ivan Guidolin Veiga ${ }^{7}$ \\ Wagner Pasqualini ${ }^{8}$ \\ Elcio Landim 9
}

\section{RESUMO}

Objetivo: avaliar os resultados clínicos e radiográficos pós-operatórios da correção de cifose congênita em pacientes com mielomeningocele de nível torácico, utilizando a prótese vertical expansível de titânio para costela (VEPTR). Métodos: estudo retrospectivo de 19 pacientes com mielomeningocele torácica e cifose congênita submetidos a tratamento com VEPTR, entre Outubro de 2005 e Outubro de 2008, com avaliação radiográfica e clínica pré e pós-operatória imediata. Foram avaliadas também a duração do proce-

\section{ABSTRACT}

Objective: to evaluate clinical and radiographic postoperative results of congenital kyphosis correction in thoracic meningomyelocele patients using vertical expandable prosthetic of titanium for the rib (VEPTR). Methods: a retrospective study of 19 thoracic meningomyelocele and congenital kyphosis patients that were subjected to the VEPTR treatment between October 2005 and October 2008, with radiographic evaluation and immediate post and pre-operative clinical practice. Also, the duration of surgical procedure, the need for

\section{RESUMEN}

Objetivo: evaluar los resultados clínicos y radiográficos postoperatorios de la corrección de la cifosis congénita en pacientes con mielomeningocele de nivel torácico, utilizando la prótesis vertical expansible de titanio para costilla (VEPTR). Métodos: estudio retrospectivo de 19 pacientes con mielomeningocele torácico y cifosis congénita sometidos al tratamiento con VEPTR, entre Octubre de 2005 y Octubre de 2008, con evaluación radiográfica y clínica, pre y postoperatorio inmediato. Fueron evaluadas también la duración del procedimiento qui-

\footnotetext{
Trabalho realizado no Grupo de Escoliose da Associação de Assistência à Criança Deficiente - AACD - São Paulo (SP), Brasil.

'Estagiário de Coluna do Departamento de Ortopedia e Traumatologia da Universidade Estadual de Campinas - UNICAMP - Campinas (SP), Brasil.

${ }^{2}$ Cirurgião de Coluna do Grupo de Escoliose da Associação de Assistência à Criança Deficiente - AACD e do Departamento de Ortopedia e Traumatologia da Universidade Estadual de Campinas - UNICAMP - Campinas (SP), Brasil.

${ }^{3}$ Cirurgião de Coluna do Grupo de Escoliose da Associação de Assistência à Criança Deficiente - AACD - São Paulo (SP), Brasil.

${ }^{4}$ Cirurgião de Coluna do Grupo de Escoliose da Associação de Assistência à Criança Deficiente - AACD - São Paulo (SP), Brasil.

${ }^{5}$ Cirurgião de Coluna do Grupo de Escoliose da Associação de Assistência à Criança Deficiente - AACD - São Paulo (SP), Brasil.

${ }^{6}$ Cirurgião de Coluna do Departamento de Ortopedia e Traumatologia da Universidade Estadual de Campinas - UNICAMP - Campinas (SP), Brasil.

${ }^{7}$ Cirurgião de Coluna do Departamento de Ortopedia e Traumatologia da Universidade Estadual de Campinas - UNICAMP - Campinas (SP), Brasil.

${ }^{8}$ Cirurgião de Coluna do Departamento de Ortopedia e Traumatologia da Universidade Estadual de Campinas - UNICAMP - Campinas (SP), Brasil.

${ }^{9}$ Chefe do Grupo de Escoliose da Associação de Assistência à Criança Deficiente - AACD e Professor do Departamento de Ortopedia e Traumatologia da Universidade Estadual de Campinas - UNICAMP - Campinas (SP), Brasil.
} 
dimento cirúrgico, a necessidade de transfusão sanguínea e as complicações pós-operatórias. Resultados: a média de idade dos pacientes foi de 70 meses ou cinco anos e dez meses (32 a 130 meses). A média de seguimento dos pacientes foi de 13,5 meses ( 2 a 26 meses). A duração média do procedimento foi de 117 minutos (variação de 70 a 195 minutos). Todas as crianças adquiriram equilíbrio de tronco, sendo que 13 delas não apresentavam isto no pré-operatório. A média da cifose pré-operatória foi de $115^{\circ}\left(80^{\circ}\right.$ a $\left.150^{\circ}\right)$ e pós-operatória de $77^{\circ}\left(50^{\circ}\right.$ a $\left.104^{\circ}\right)$, com porcentagem média de correção de $31,2 \%$ $(1,1$ a $61,5 \%)$. O desequilíbrio do tronco pré-operatório foi de $7,9 \mathrm{~cm}$, em média ( 1,0 a $15,5 \mathrm{~cm})$ e pós-operatório de $3,4 \mathrm{~cm}(0$ a $8 \mathrm{~cm})$. A correção média desse desequilíbrio foi de $50,4 \%$ (0 a 100\%). Com relação ao peso, no pré-operatório a média foi de $15,4 \mathrm{~kg}$ ( 8 a $30 \mathrm{~kg}$ ), e no pósoperatório de $20,6 \mathrm{~kg}(8,5 \mathrm{a} 35 \mathrm{~kg})$. O ganho médio de peso foi de $36,6 \%$ $(9,8$ a $100 \%)$. Dos 19 pacientes, cinco $(26,3 \%)$ apresentaram complicações pós-operatórias. Nenhum paciente necessitou de transfusão sanguínea. Conclusão: a utilização do VEPTR nos pacientes portadores de mielomeningocele torácica com cifose congênita tem se mostrado uma alternativa eficaz e promissora de controle da deformidade em pacientes esqueleticamente imaturos. blood transfusion and postoperative complications were assessed. Results: the patients' average age was 70 months (from 32 to 130 months). The average follow-up from patients was 13.5 months (from 2 to 26 months). The average duration of the procedure was 117 minutes (variation between 70 and 195 minutes). All children reached trunk balance, 13 of whom had not showed it in the postoperative period. The average of pre-operation kyphosis was $115^{\circ}$ (from $80^{\circ}$ to $150^{\circ}$ ) and $77^{\circ}$ (from $50^{\circ}$ to $104^{\circ}$ ) for postoperative, with an average correction percentage of $31.2 \%$ (from 1.1 to $61.5 \%)$. The previous preoperative imbalance of trunk was an average $7.9 \mathrm{~cm}$ (from 1.0 to $15.5 \mathrm{~cm}$ ) and $3.4 \mathrm{~cm}$ (from 0 to $8 \mathrm{~cm}$ ) for postoperative. The average correction of this imbalance was of $50.4 \%$ (from 0 to $100 \%$ ). Regarding weight, in pre-operative the average was 15.4 $\mathrm{kg}$ (from 8 to $30 \mathrm{~kg}$ ) and $20.6 \mathrm{~kg}$ (from 8.5 to $35 \mathrm{~kg}$ ) for postoperative. The average gain of weight was of $36.6 \%$ (from 9.8 to $100 \%$ ). Five of the 19 patients $(26.3 \%)$ presented postoperative complications. No patient needed blood transfusion. Conclusion: the use of VEPTR in thoracic meningomyelocele and congenital kyphosis patients has proven to be an effective and promising alternative for the control of physical deformity in patients with a potential for growth. rúrgico, la necesidad de transfusión sanguinea y las complicaciones postoperatorias. Resultados: el promedio de edad de los pacientes fue de 70 meses o 5 años y 10 meses (32 a 130 meses). El promedio de seguimiento de los pacientes fue de 13.5 meses ( 2 a 26 meses). La duración promedio del procedimiento fue de 117 minutos ( $v a-$ riación de 70 a 195 minutos). Todos los niños adquirieron equilibrio del tronco, siendo que 13 no lo presentaban en el preoperatorio. El promedio de la cifosis preoperatorio fue de $115^{\circ}$ $\left(80^{\circ}\right.$ a $\left.150^{\circ}\right)$ y postoperatoria de $77^{\circ}$ $\left(50^{\circ}\right.$ a $\left.104^{\circ}\right)$, con porcentaje promedio de corrección de $31.2 \%$ (1.1 a 61.5\%). El desequilibrio del tronco preoperatorio fue de $7.9 \mathrm{~cm}$, en promedio $(1.0$ a $15.5 \mathrm{~cm}$ ) y postoperatorio de $3.4 \mathrm{~cm}$ (0 a $8 \mathrm{~cm}$ ). La corrección promedio de ese desequilibrio fue de $50.4 \%$ (0 a 100\%). Con relación al peso, en el preoperatorio el promedio fue de 15.4 $\mathrm{kg}$ ( 8 a $30 \mathrm{~kg}), y$ en el postoperatorio de $20.6 \mathrm{~kg}(8.5$ a $35 \mathrm{~kg})$. La ganancia de peso en promedio fue de $36.6 \%(9.8$ a 100\%). De los 19 pacientes, cinco (26.3\%) presentaron complicaciones postoperatorias. Ningún paciente necesitó transfusión sanguínea. Conclusión: la utilización del VEPTR en los pacientes portadores de mielomeningocele torácico con cifosis congénita ha demostrado ser una alternativa eficaz y promisoria del control de la deformidad en pacientes esqueléticamente inmaduros.
DESCRITORES: Cifose/congênito; Meningomielocele/ instrumentação; Próteses; Titânio
KEYWORDS: Kyphosis/congenital; Meningomyelocele/ instrumentation; Prostheses; Titaniun
DESCRIPTORES: Cifosis/ congénito; Meningomielocele/ instrumentación; Prótesis e implantes; Titanio

\section{INTRODUÇÃO}

A cifose nos pacientes portadores de mielomeningocele é causa de muitas complicações clínicas desde o nascimento. Os pacientes apresentam uma grande protuberância em ângulo agudo na face posterior do tronco ${ }^{1}$, a falta de sensibilidade e a pressão exercida na pele sobre essa região provocam ulcerações causando desde infecções locais até meningite $^{2}$. Comprometimento respiratório ${ }^{3}$ e problemas com a deglutição e digestão ${ }^{4}$ ocorrem pelo aumento da pressão do conteúdo abdominal contra o diafragma, fazendo com que sejam crianças de baixo peso. A flexão aumentada do tronco também pode interferir na drenagem urinária, provocando infecções urinárias de repetição o que deteriora a função renal (Figura 1).

Nos pacientes com mielomeningocele existem dois tipos de cifose: paralítica por insuficiência muscular e as congênitas ${ }^{5}$. A cifose nos portadores de mielomeningocele tem incidência que varia entre $1^{6}$ a $46 \%{ }^{7}$, e a forma 
congênita corresponde a $13,9 \%{ }^{8}$. A cifose congênita é causada por uma falha de formação ou de segmentação de pelo menos uma vértebra, sendo mais frequente a falha de formação da porção anterior do corpo vertebral ${ }^{9,10}$. São deformidades de raio curto, com ápice geralmente entre T12 e L1 e se estende até a articulação L5-S1. Tal deformidade está presente desde o nascimento e tende a progredir mesmo após a maturidade esquelética ${ }^{1}$, com progressão anual média de $8,3^{\circ 11}$. A progressão da deformidade representa agravamento das condições respiratórias, nutricionais, psicológicas, urinárias e de pele.

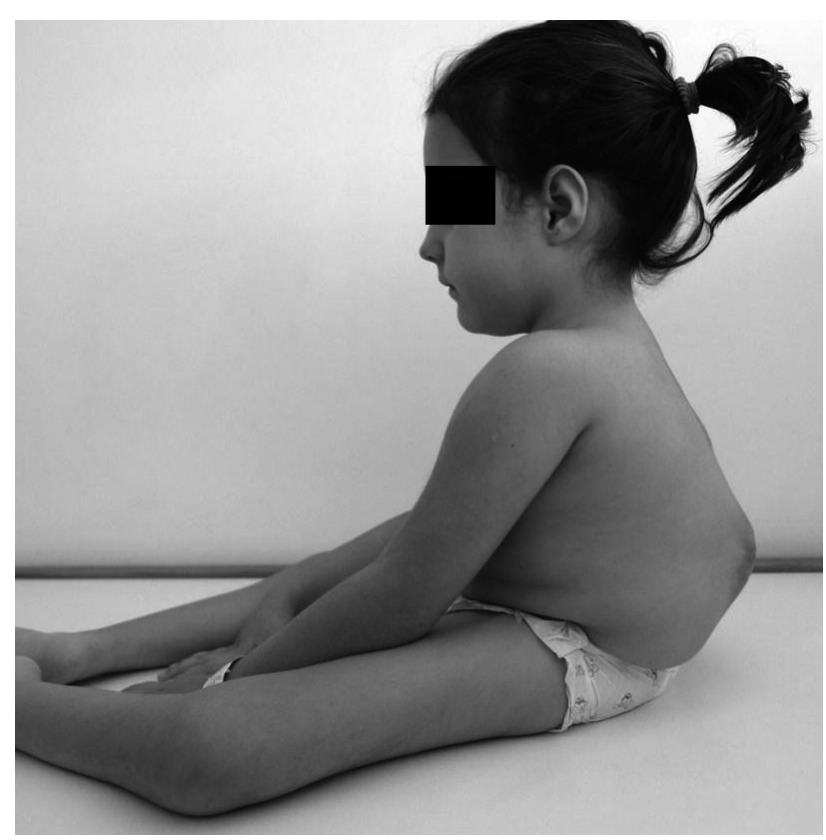

Figura 1

Aspecto clínico de criança com mielomeningocele e cifose congênita.

A cifose na mielomeningocele é mantida e agravada pelo deslocamento anterior da musculatura espinhal extensora que passa a atuar como flexora da coluna lombar ${ }^{12} \mathrm{e}$, além disso, o músculo psoas hipertrofiado e a inserção do pilar do diafragma na vértebra apical também provocam flexão da coluna lombar ${ }^{13}$. Nessas crianças, o ligamento longitudinal anterior está encurtado e a porção anterior do ânulo fibroso tensa, com o núcleo pulposo deslocado para trás ${ }^{1}$. A pelve está rodada para frente ${ }^{12}$.

A deformidade altera o centro de gravidade do tronco, as crianças com cifose congênita costumam se sentar com idade mais avançada, em comparação a crianças normais, e o peso do corpo na posição sentada pode contribuir para a progressão da cifose ${ }^{1}$. Junto à cifose há uma lordose compensatória prolongando-se às vértebras torácicas que alguns autores acreditam estar presente desde o nascimento ${ }^{14}$. Outros autores a descrevem como uma deformidade compensatória progressiva a partir do momento em que a criança começa a sentar ${ }^{1,12}$. O desequilíbrio anterior do tronco pela flexão contínua do tronco obriga a utilização das mãos para apoio e manutenção da postura na posição sentada ${ }^{15}$ (Figura 2).

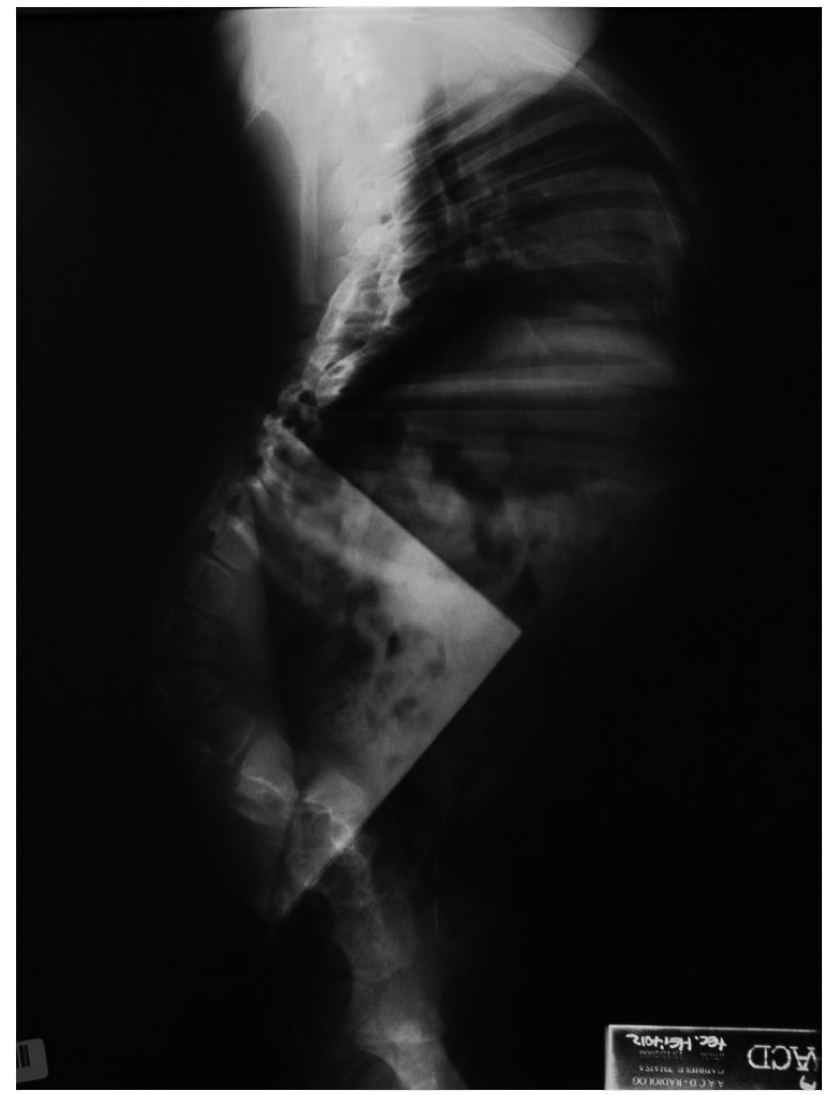

Figura 2

Aspecto radiográfico: cifose de raio curto, lordose torácica compensatória e anteriorização do tronco.

O tratamento com órteses nesses pacientes é ineficaz e seu uso é contraindicado ${ }^{16}$. O tratamento cirúrgico das deformidades vertebrais nos pacientes com mielomeningocele torácica tem como objetivo melhorar o equilíbrio sentado, diminuir a incidência de úlceras de pressão e impedir a progressão da deformidade ${ }^{17}$. Os pacientes apresentam baixo peso e estatura, tornando a cirurgia um desafio. Além disso, a hipoplasia, o deslocamento dos elementos vertebrais posteriores e a osteopenia generalizada dificultam a instrumentação ${ }^{15,18}$.

As indicações de tratamento cirúrgico costumam ser ulceração crônica, dificuldade para sentar, progressão da deformidade e impossibilidade de manter a criança em decúbito dorsal ${ }^{15}$. Além das indicações, o tratamento cirúrgico ainda apresenta algumas controvérsias em relação a técnicas, métodos de fixação e idade ideal para a realização do procedimento. As técnicas cirúrgicas descritas até o momento envolvem ressecções vertebrais, variando quanto à extensão da artrodese: curta, para crianças de menor idade e, longa para crianças de maior idade ${ }^{4}$. A artrodese curta tem como inconveniência a necessidade de imobilização pós-operatória ${ }^{12}$ e a artrodese longa, por sua vez, impede o crescimento longitudinal do tronco. A cirurgia ideal, num paciente com imaturidade óssea, permitiria a preservação do potencial de crescimento da coluna, promoveria uma boa correção da deformidade com melhora 
das condições clínicas, diminuindo, assim, as complicações decorrentes da deformidade.

Há algumas décadas, técnicas de correção de deformidades da coluna sem artrodese estão se desenvolvendo $^{19}$. A prótese vertical expansível de titânio para costela (VEPTR ${ }^{\circledR}$; Synthes Spine Co, West Chester, Pennsylvania, USA) é um sistema que permite alongamentos longitudinais seriados. Seu uso foi aprovado pela Food and Drug Administration (FDA) para uso na Síndrome de Insuficiência Torácica (SIT) ${ }^{20}$. Nos últimos anos, seu uso tem se expandido para o tratamento de outras deformidades da coluna vertebral, como escoliose congênita e neuromuscular em pacientes com imaturidade óssea. Nesse estudo, o VEPTR foi utilizado no tratamento da cifose congênita em pacientes com mielomeningocele.

O objetivo desse estudo é avaliar os resultados clínicos e radiográficos pós-operatórios de pacientes portadores de mielomeningocele torácica com cifose congênita tratados com VEPTR, bem como a duração e necessidade de transfusão sanguínea do procedimento.

\section{MÉTODOS}

Foi realizado estudo retrospectivo em 19 pacientes portadores de mielomeningocele e cifose congênita submetidos ao tratamento cirúrgico com VEPTR, entre Outubro de 2005 e Outubro de 2008. Todos os casos foram operados pelo Grupo de Escoliose da Associação de Assistência à Criança Deficiente (AACD) em São Paulo. O tratamento com VEPTR foi indicado para crianças com potencial de crescimento e/ou baixo peso para uma cirurgia de vertebrectomia e artrodese. Todos os procedimentos foram realizados em ambiente hospitalar com anestesia geral e em decúbito ventral. Na montagem do sistema, o pós-operatório foi realizado em Unidade de Terapia Intensiva (UTI) com fisioterapia respiratória. Os alongamentos não necessitaram de cuidados pós-operatórios em UTI. As crianças apresentavam exames laboratoriais sanguíneos normais (hemograma e coagulograma) e urocultura negativa na internação. Em todos os casos de montagem e alongamento realizou-se profilaxia antibiótica, de acordo com o padrão estabelecido pela Comissão de Controle de Infecção Hospitalar (CCIH). Todos apresentavam também teste de alergia ao látex.

As montagens foram feitas com duas hastes paramedianas (direita e esquerda), fixadas na costela e na pelve, por quatro incisões longitudinais: duas distais (paravertebral próximo ao ápice da crista ilíaca) e duas proximais (paraescapular na altura da costela em que se realizaria a colocação dos ganchos). A montagem da porção distal do VEPTR necessitou descolamento da apófise da crista ilíaca para introdução do gancho no ilíaco e, da porção proximal, descolamento das partes moles, com preservação do periósteo da costela escolhida. As duas hastes ficaram paralelas entre si e perpendiculares ao solo para evitar o desequilíbrio lateral durante o crescimento e/ou alongamentos. A colocação do implante no plano submuscular e o fechamento por planos, buscando a maior cobertura com tecido mole, objetiva evitar saliência do implante ou ulcerações da pele. O uso de dreno de sucção não foi necessário em nenhum caso (Figuras 3, 4 e 5).

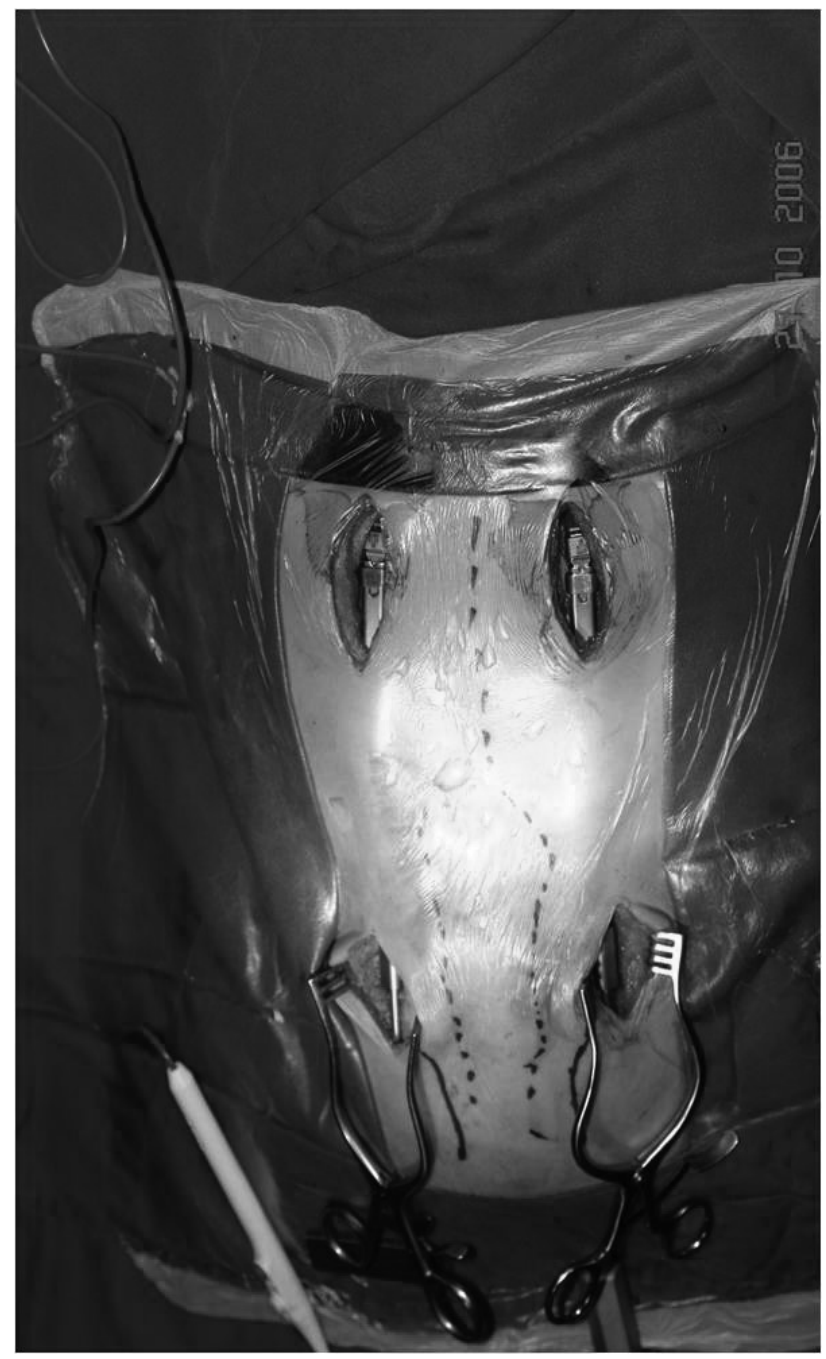

Figura 3

Vias de acesso para montagem do sistema.

Os alongamentos, necessários para acompanhar o crescimento do tronco, são realizados por meio de incisões longitudinais ou transversas sobre as travas identificadas pela palpação. O período entre os alongamentos varia de quatro a seis meses.

Em nenhum caso, utilizou-se imobilização pós-operatória e o programa de reabilitação foi mantido, com orientação apenas para evitar atividades com flexão excessiva do tronco.

As variáveis clínicas analisadas foram: peso e equilíbrio do tronco pré e pós-operatório e as complicações decorrentes tanto do procedimento de montagem como de alongamento do sistema. Foram também comparadas as radiografias pré- e pós-operatória com avaliação do desvio no plano sagital (cifose), pelo método de Cobb, além do desequilíbrio anterior do tronco, medido como a distância entre a porção póstero-superior da primeira vértebra sacral 


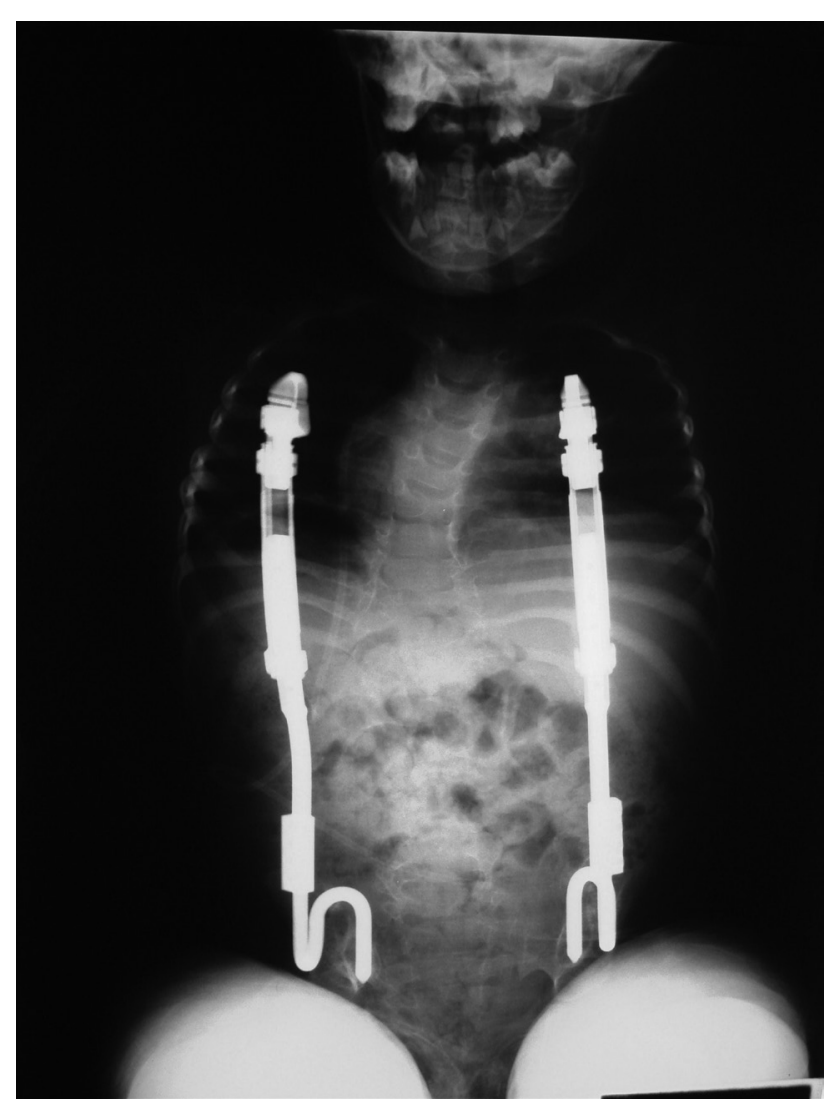

Figura 4

Radiografia pós-operatória em AP.

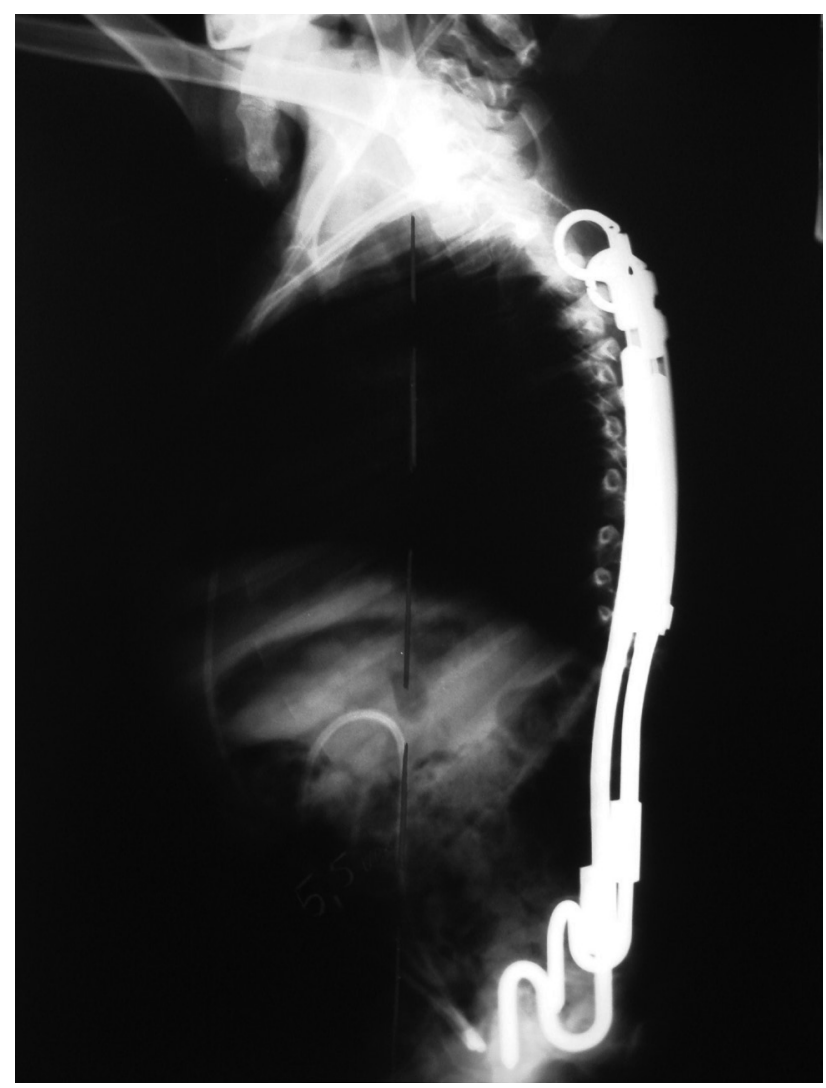

Figura 5

Radiografia pós-operatória em perfil. e uma linha perpendicular ao solo a partir do centro do corpo da sétima vértebra cervical. $\mathrm{O}$ estudo também avaliou a duração e a necessidade de transfusão sanguínea durante os procedimentos de montagem do sistema. A análise estatística foi feita por meio de teste $t$ não pareado.

\section{RESULTADOS}

Foram submetidas a tratamento cirúrgico entre Outubro de 2005 e Outubro de 2008, 19 crianças, sendo 12 do sexo feminino e 7 do sexo masculino. A média de idade foi de 70 meses ou 5 anos e 10 meses, variação de 32 a 130 meses com desvio padrão de 23,5 meses. A média de seguimento dos pacientes foi de 13,5 meses (variação de 2 a 26 meses, com desvio padrão de 5,6 meses). A duração média do procedimento de montagem foi de 117 minutos (variação de 70 a 195 minutos e desvio padrão de 35 minutos). O período de internação para montagem do VEPTR foi de em média 5,3 dias, com variação de 4 a 16 dias (desvio padrão de 2,7 dias).

Treze dos 19 pacientes já haviam sido submetidos ao menos um procedimento para alongamento. Dentre essas crianças, uma foi alongada quatro vezes, duas, a três cirurgias, sete, a dois alongamentos e, as outras três, a um procedimento cada uma, totalizando 27 procedimentos para alongamento. Os alongamentos duram cerca de 20 minutos, com alta hospitalar no dia seguinte ao procedimento (Tabela 1).

A média da cifose pré-operatória foi de $115^{\circ}$ (variação de $80^{\circ}$ a $150^{\circ}$, desvio padrão de $23^{\circ}$ ), e pós-operatória de $77^{\circ}$ (variação de $50^{\circ}$ a $104^{\circ}$, desvio padrão de $16^{\circ}$ ) com porcentagem média de correção de 31,2\% (variação de 1,1 a $61,5 \%$ e desvio padrão de $15,9 \%$ ). Houve diferença estatisticamente significante $(\mathrm{p}<0,0001)$ entre as médias do valor da cifose pré e pós-operatórias. $\mathrm{O}$ desequilíbrio do tronco pré-operatório foi de 7,9 cm, em média, com variação de 1,0 a $15,5 \mathrm{~cm}$ (desvio padrão de $3,5 \mathrm{~cm}$ ) e pósoperatório de $3,4 \mathrm{~cm}$, com variação de 0 a $8 \mathrm{~cm}$ (desvio padrão de 2,2 cm). Também houve diferença estatisticamente significante entre as médias pré e pós-operatórias $(\mathrm{p}<0,0001)$. A correção média desse desequilíbrio foi de 50,4\% (variação de 0 a $100 \%$, desvio padrão de 33,4\%). Treze crianças que não apresentavam equilíbrio do tronco no período pré-operatório adquiriram essa capacidade no pós-operatório (Tabela 2).

Com relação ao peso, no pré-operatório a média foi de $15,4 \mathrm{~kg}$, variando de 8 a $30 \mathrm{~kg}$ (desvio padrão de 5,3 kg), e no pós-operatório de $20,6 \mathrm{~kg}$, com variação de 8,5 a 35 $\mathrm{kg}$ (desvio padrão de $6,4 \mathrm{~kg}$ ). Nenhuma criança teve seu peso reduzido no seguimento pós-operatório. O ganho médio de peso foi de $36,6 \%$ (variação de 9,8 a $100 \%$, desvio padrão de $21,7 \%$ ). A diferença entre os pesos no pré e pósoperatório foi estatisticamente significante $(p=0,0097)$. Nove crianças mudaram seu percentil de peso nas curvas de acompanhamento do peso de acordo com a idade. Os outros dez pacientes, apesar do ganho de peso, mantiveram-se dentro do percentil de peso do pré-operatório. A 
TABELA 1 - Sexo, idade na época de cirurgia, seguimento e número de alongamentos

\begin{tabular}{lcccc}
\hline Caso & Sexo & Idade & Seguimento & Alongamentos \\
\hline 1 & $M$ & 3 a $8 \mathrm{~m}$ & $26 \mathrm{~m}$ & 4 \\
2 & $\mathrm{~F}$ & $6 \mathrm{a} 3 \mathrm{~m}$ & $17 \mathrm{~m}$ & 0 \\
3 & $\mathrm{~F}$ & $3 \mathrm{a}$ & $16 \mathrm{~m}$ & 2 \\
4 & $\mathrm{M}$ & $5 \mathrm{a} 3 \mathrm{~m}$ & $17 \mathrm{~m}$ & 2 \\
5 & $\mathrm{M}$ & $6 \mathrm{a} 2 \mathrm{~m}$ & $18 \mathrm{~m}$ & 1 \\
6 & $\mathrm{~F}$ & $6 \mathrm{a} 11 \mathrm{~m}$ & $16 \mathrm{~m}$ & 2 \\
7 & $\mathrm{~F}$ & $6 \mathrm{a} 0 \mathrm{~m}$ & $17 \mathrm{~m}$ & 1 \\
8 & $\mathrm{~F}$ & $6 \mathrm{a} 10 \mathrm{~m}$ & $13 \mathrm{~m}$ & 3 \\
9 & $\mathrm{~F}$ & $7 \mathrm{a} 2 \mathrm{~m}$ & $19 \mathrm{~m}$ & 2 \\
10 & $\mathrm{~F}$ & $10 \mathrm{a} 10 \mathrm{~m}$ & 1 \\
11 & $\mathrm{M}$ & $7 \mathrm{a} 10 \mathrm{~m}$ & $14 \mathrm{~m}$ & 2 \\
12 & $\mathrm{~F}$ & $4 \mathrm{a} 11 \mathrm{~m}$ & $10 \mathrm{~m}$ & 0 \\
13 & $\mathrm{~F}$ & $6 \mathrm{a} 7 \mathrm{~m}$ & $12 \mathrm{~m}$ & 0 \\
14 & $\mathrm{M}$ & $2 \mathrm{a} 8 \mathrm{~m}$ & $10 \mathrm{~m}$ & 3 \\
15 & $\mathrm{~F}$ & $4 \mathrm{a} 4 \mathrm{~m}$ & $12 \mathrm{~m}$ & 0 \\
16 & $\mathrm{M}$ & $8 \mathrm{a}$ & $9 \mathrm{~m}$ & 0 \\
17 & $\mathrm{~F}$ & $5 \mathrm{a} 4 \mathrm{~m}$ & $7 \mathrm{~m}$ & 0 \\
18 & $\mathrm{~F}$ & $4 \mathrm{a} 1 \mathrm{~m}$ & $2 \mathrm{~m}$ & 0 \\
19 & $\mathrm{M}$ & $4 \mathrm{a} 9 \mathrm{~m}$ & $5 \mathrm{~m}$ & \\
\hline
\end{tabular}

M: masculino; F: feminino, a: anos; m: meses.

TABELA 2 - Análise pré e pós-operatória da cifose e desequilíbrio anterior do tronco

\begin{tabular}{|c|c|c|c|c|c|c|}
\hline \multirow{2}{*}{ Caso } & \multicolumn{3}{|c|}{ Cifose } & \multicolumn{3}{|c|}{ Desequilíbrio anterior do tronco } \\
\hline & Pré & Pós & \% Correção & Pré $(\mathrm{cm})$ & Pós (cm) & \% Correção \\
\hline 1 & $108^{\circ}$ & $70^{\circ}$ & 35,2 & 5 & 1,5 & 70 \\
\hline 2 & $140^{\circ}$ & $104^{\circ}$ & 25,7 & 4,3 & 2,9 & 32,6 \\
\hline 3 & $125^{\circ}$ & $75^{\circ}$ & 40 & 9,5 & 0,4 & 95,8 \\
\hline 4 & $130^{\circ}$ & $50^{\circ}$ & 61,5 & 10,5 & 3 & 71,4 \\
\hline 5 & $150^{\circ}$ & $92^{\circ}$ & 38,7 & 10,5 & 3,7 & 64,8 \\
\hline 6 & $125^{\circ}$ & $100^{\circ}$ & 20 & 11,9 & 2 & 83,2 \\
\hline 7 & $92^{\circ}$ & $69^{\circ}$ & 25 & 7,1 & 6 & 15,5 \\
\hline 8 & $90^{\circ}$ & $89^{\circ}$ & 1,1 & 9 & 7 & 22,2 \\
\hline 9 & 33 & $82^{\circ}$ & 33,9 & 3,4 & 2,6 & 23,5 \\
\hline 10 & $89^{\circ}$ & $74^{\circ}$ & 16,9 & 9,4 & 8 & 0 \\
\hline 11 & $148^{\circ}$ & $60^{\circ}$ & 59,5 & 15,5 & 1 & 93,5 \\
\hline 12 & $80^{\circ}$ & $60^{\circ}$ & 25 & 5,6 & 5,5 & 1,8 \\
\hline 13 & $100^{\circ}$ & $58^{\circ}$ & 42 & 3,5 & 3 & 14,3 \\
\hline 14 & $123^{\circ}$ & $75^{\circ}$ & 39 & 6,3 & 4,5 & 28,6 \\
\hline 15 & $140^{\circ}$ & $75^{\circ}$ & 46,4 & 12 & 2,4 & 80 \\
\hline 16 & $130^{\circ}$ & $100^{\circ}$ & 23 & 10,8 & 2,5 & 76,9 \\
\hline 17 & $118^{\circ}$ & $81^{\circ}$ & 31,4 & 9,8 & 5 & 49 \\
\hline 18 & $85^{\circ}$ & $60^{\circ}$ & 29,4 & 3,4 & 0 & 100 \\
\hline 19 & $86^{\circ}$ & $84^{\circ}$ & 2,3 & 4,5 & 3 & 33,3 \\
\hline
\end{tabular}

velocidade média de ganho de peso no período pós-operatório foi de 506,4 g/mês, variando de 123 a $2.900 \mathrm{~g} / \mathrm{mês}$, com desvio padrão de 598,4 g/mês (Tabela 3).

Dos 19 pacientes, quatro $(21,0 \%)$ apresentaram complicações relacionadas com o procedimento após a montagem do sistema e uma (5,3\%) apresentou uma complicação inespecífica (transtorno de estresse pós-cirúrgico). As complicações específicas foram infecção (2), infecção com soltura do implante (1) e fratura de costela (1). Três infecções $(11,1 \%)$ ocorreram após os procedimentos de alongamento. Em um dos casos de infecção após alongamento, foi necessário retirar uma das hastes 
TABELA 3 - Avaliação pré-operatória e atual do peso com velocidade de ganho de peso e percentil de acordo com sexo e idade

\begin{tabular}{|c|c|c|c|c|c|c|}
\hline \multirow{2}{*}{ Caso } & \multicolumn{4}{|c|}{ Peso } & \multicolumn{2}{|c|}{ Percentil } \\
\hline & Pré $(\mathrm{kg})$ & Pós (kg) & $\%$ ganho & Vel. ganho & Pré & Pós \\
\hline 1 & 11,8 & 15 & 27,1 & 123 & $<\mathrm{p} 5$ & $<\mathrm{p} 5$ \\
\hline 2 & 14 & 21 & 50 & 411,8 & $<\mathrm{p} 5$ & p $10-25$ \\
\hline 3 & 8 & 11,5 & 43,8 & 218,8 & $<\mathrm{p} 5$ & $<\mathrm{p} 5$ \\
\hline 4 & 10 & 20 & 100 & 588,2 & $<\mathrm{p} 5$ & p25-50 \\
\hline 5 & 19 & 23 & 21 & 222,2 & p25 & p50 \\
\hline 6 & 22 & 30 & 36,4 & 500 & p50 & p75 \\
\hline 7 & 17 & 26 & 52,9 & 529,4 & p25 & p75 \\
\hline 8 & 30 & 35 & 16,7 & 384,6 & p90-95 & p90-95 \\
\hline 9 & 10,7 & 15,3 & 43 & 242,1 & $<\mathrm{p} 5$ & $<\mathrm{p} 5$ \\
\hline 10 & 14,5 & 21 & 44,8 & 464,3 & $<p 5$ & $<\mathrm{p} 5$ \\
\hline 11 & 14 & 17,8 & 27,1 & 380 & $<\mathrm{p} 5$ & $<\mathrm{p} 5$ \\
\hline 12 & 13 & 15 & 15,4 & 166,7 & $<\mathrm{p} 5$ & $<\mathrm{p} 5$ \\
\hline 13 & 12,5 & 15,2 & 21,6 & 270 & $<\mathrm{p} 5$ & $<\mathrm{p} 5$ \\
\hline 14 & 24 & 30 & 25 & 500 & $>$ p95 & $>$ p95 \\
\hline 15 & 12,9 & 16,5 & 27,9 & 400 & $<\mathrm{p} 5$ & p25-50 \\
\hline 16 & 17 & 28 & 64,7 & 687,5 & $<\mathrm{p} 5$ & p50 \\
\hline 17 & 13,8 & 16 & 15,9 & 314,3 & $<\mathrm{p} 5$ & p5 \\
\hline 18 & 11,2 & 17 & 51,8 & 2900 & $<\mathrm{p} 5$ & p50 \\
\hline 19 & 16,4 & 18 & 9,8 & 320 & p25 & p50 \\
\hline
\end{tabular}

p: percentil; vel. ganho: velocidade de ganho de peso (gramas/mês).

com seus ganchos, pois não houve resposta ao desbridamento cirúrgico e antibioticoterapia; outro apresentou boa resposta ao tratamento clínico (antibiótico, oxigenoterapia hiperbárica e cuidados locais) e, no último, foi necessário realizar o desbridamento cirúrgico, com boa evolução no seguimento. Nos dois casos em que ocorreram infecção e soltura do implante, a soltura ocorreu na porção distal da montagem (fixação no ilíaco). Ambos foram tratados com reposicionamento do implante, por ocasião da cirurgia para alongamento, desbridamento e antibioticoterapia, com boa resolução dos casos. No caso em que foi apresentada fratura de costela, realizou-se o reposicionamento da montagem numa costela proximal àquela fraturada, com consolidação da fratura e sem novas solturas. $\mathrm{O}$ transtorno de estresse pós-cirúrgico foi acompanhado por um psicólogo, tendo evolução satisfatória e com o paciente já tendo sido submetido, inclusive, a dois alongamentos desde então. Nenhum paciente necessitou de transfusão sanguínea durante a cirurgia de montagem ou alongamento e nem durante as internações (Tabela 4, Figuras 6, 7 e 8).

\section{DISCUSSÃO}

A mielomeningocele é uma malformação de tratamento complexo e multidisciplinar, devendo ser realizada em centros especializados. Os avanços da neurocirurgia e urologia aumentaram a sobrevida das crianças com mielomeningocele, aumentando, assim, a quantidade de crianças com deformidades ortopédicas da coluna vertebral. A cifose congênita nesses pacientes é uma deformidade de caráter progressivo. Banta e Hamada ${ }^{11}$ relatam uma progressão anual média de

\section{TABELA 4 - Duração da montagem do sistema} e complicações

\begin{tabular}{|c|c|c|}
\hline Caso & $\begin{array}{l}\text { Tempo de cirurgia } \\
\text { (minutos) }\end{array}$ & Complicação \\
\hline 1 & 140 & Não \\
\hline 2 & 80 & Não \\
\hline 3 & 120 & Não \\
\hline 4 & 100 & Infecção \\
\hline 5 & 100 & Não \\
\hline 6 & 150 & Infecção \\
\hline 7 & 90 & Infecção + soltura \\
\hline 8 & 195 & Infecção \\
\hline 9 & 105 & Não \\
\hline 10 & 165 & Não \\
\hline 11 & 150 & Infecção + soltura \\
\hline 12 & 100 & Não \\
\hline 13 & 120 & Não \\
\hline 14 & 100 & Estresse pós-cirúrgico \\
\hline 15 & 70 & Não \\
\hline 16 & 120 & Soltura - fratura de costela \\
\hline 17 & 80 & Não \\
\hline 18 & 90 & Não \\
\hline 19 & 80 & Infecção \\
\hline
\end{tabular}




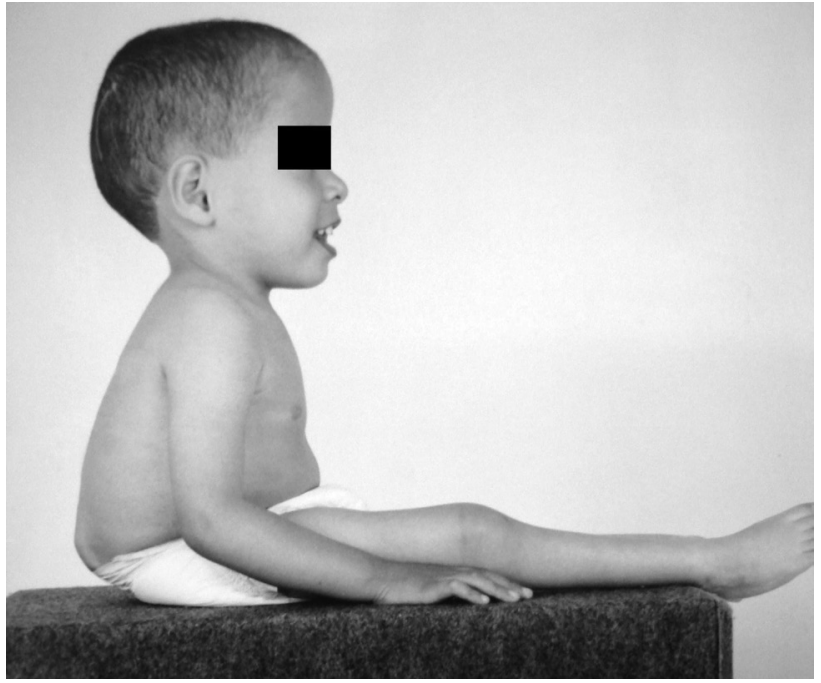

Figura 6

Aspecto clínico pré-operatório.

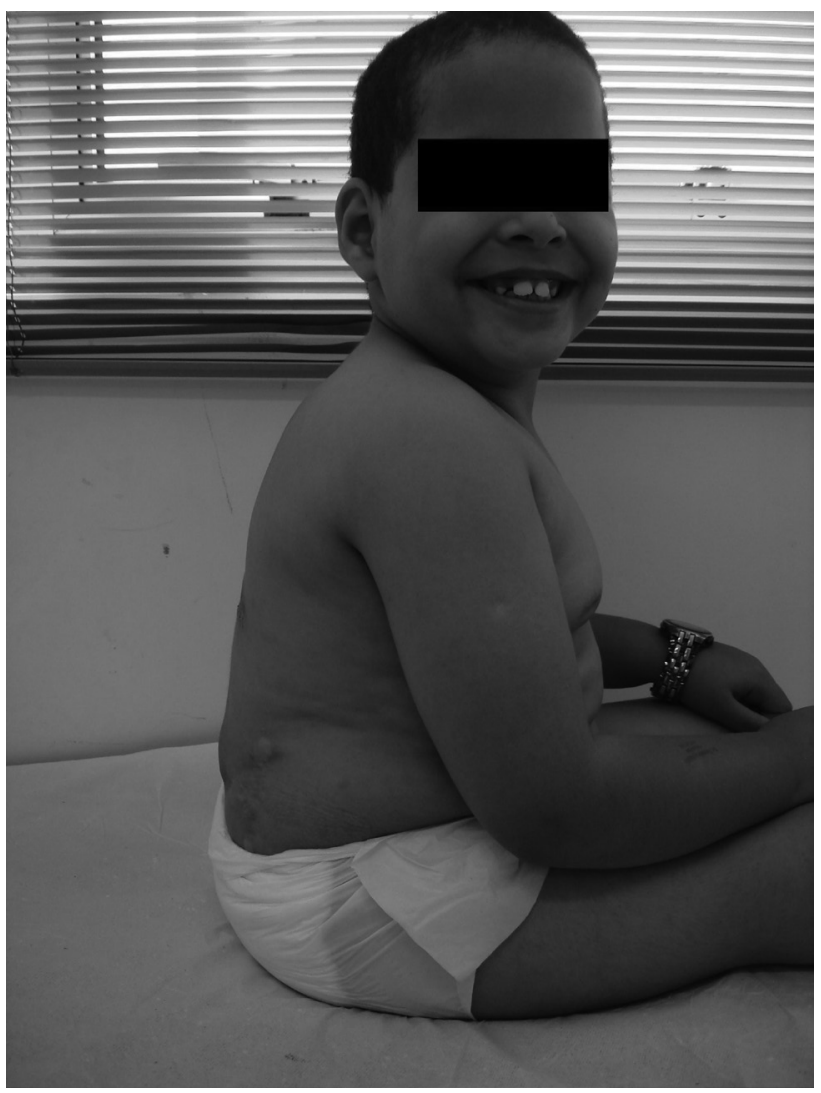

Figura 7

Aspecto clínico após 16 meses de seguimento com três alongamentos.

$8,3^{\circ}$, valor bastante parecido com os encontrados por Sarwark $^{21}$ de $8^{\circ}$. Mintz et al. ${ }^{22}$ descrevem uma média de progressão anual de $7,7^{\circ}$ se a cifose inicial for menor que $90^{\circ}$, e de $12,1^{\circ}$ se a deformidade inicial for maior que $90^{\circ}$.

Sharrard $^{12}$, em 1968, descreveu a primeira técnica de ressecção vertebral com fusão com grampos de Blount apenas na área de osteotomia para correção da cifose congênita nos recém-nascidos com mielomeningocele. O

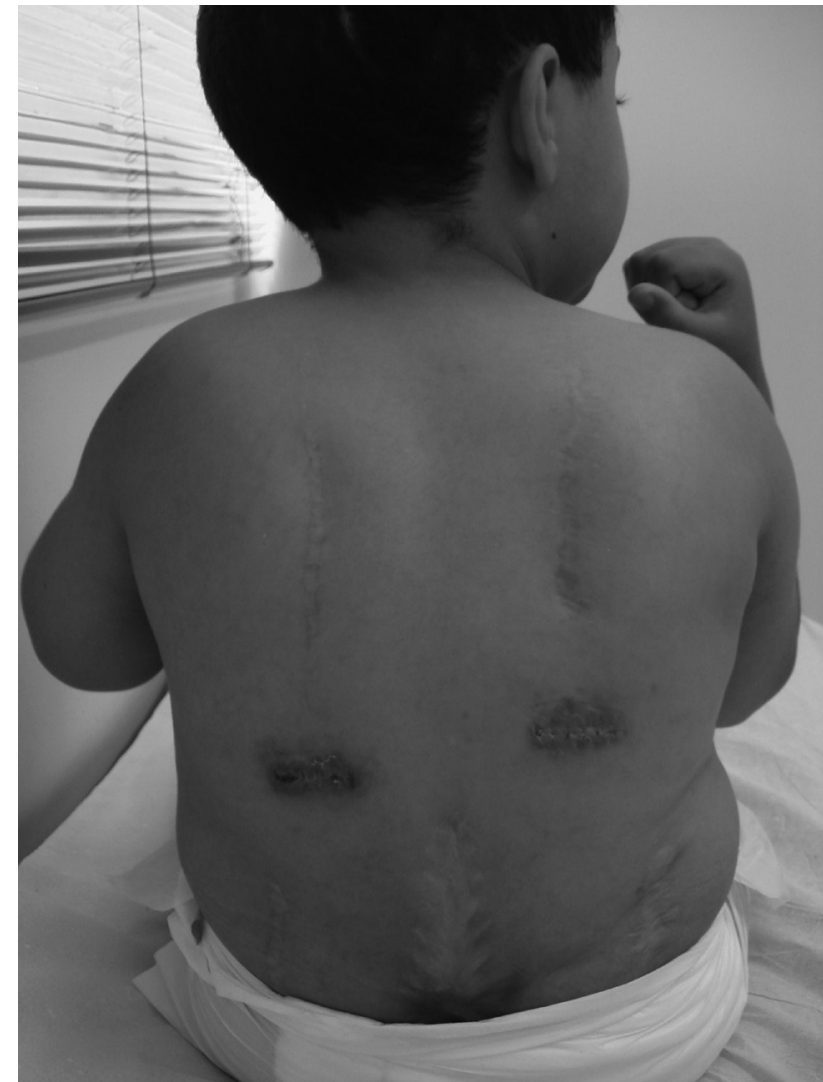

Figura 8

Visão posterior com cicatrizes da montagem e dos alongamentos.

procedimento apresentou uma alta mortalidade (três mortes em seis crianças operadas $)^{23}$. Sharrard e Drennan ${ }^{23}$ observaram uma progressão de $15^{\circ}$ nos primeiros dois anos de vida dos pacientes tratados cirurgicamente contra $45^{\circ}$ de progressão nas crianças não operadas. Desde então, outras vias de acesso, técnicas de instrumentação para diferentes faixas etárias, cifosectomia e correção da curva têm sido descritas $8,10,14,16,24-31$.

O foco do tratamento é o controle da curva pela artrodese. Tal controle é obtido pelo restabelecimento completo do equilíbrio sagital ${ }^{27,32}$, estabilizando a coluna sobre a bacia $^{33}$, garantindo, assim, a durabilidade da correção $0^{21}$. A idade ideal para o tratamento cirúrgico, o método de correção da deformidade, a extensão e o tipo da instrumentação são pontos ainda a serem definidos ${ }^{29}$.

Nenhuma das técnicas tem se mostrado superior ${ }^{18}$. McMaster $^{26}$, por exemplo, acredita que a idade ideal para correção da deformidade seja entre oito e dez anos, já que tem como princípio de tratamento artrodeses longas, que, em crianças pequenas acarretariam encurtamento do tronco e, em adolescentes, a deformidade já seria grave o bastante, impedindo uma correção satisfatória. Lowe e Menelaus $^{27}$ têm como idade ideal entre três e seis anos para realização de excisão vertebral apical e fusão extensa com fios rosqueados para correção tanto da cifose como das deformidades compensatórias. Hall e Poitras ${ }^{34}$ também preferem a cirurgia após os três anos, pois nas crianças 
abaixo dessa idade o índice de perda de correção é muito elevado pela dificuldade de estabilização e instrumentação das vértebras. No entanto, os defensores do tratamento cirúrgico precoce alegam que o objetivo é diminuir a velocidade de progressão da deformidade, diminuindo as ulcerações de pele, osteomielite e septicemia, evitando o aparecimento de deformidades compensatórias ${ }^{1}$, e facilitando o fechamento de pele na correção da mielomeningocele $^{12}$. Acreditam também que a cirurgia em idade precoce pode estar associada a melhores resultados a longo prazo ${ }^{35}$.

Como são procedimentos cirúrgicos de grande porte em pacientes com problemas clínicos como infecções urinárias de repetição e úlceras de pressão e, que apresentam pele sem sensibilidade e com cicatriz extensa dificultando o fechamento pós-operatório, observa-se altas taxas de complicações precoces e tardias, inclusive morte por sangramento ou alteração da dinâmica do líquido cefalorraquidiano ${ }^{24}$. Outras complicações também foram descritas nesses procedimentos: osteomielite ${ }^{2}$, meningite $^{2}$, perda da correção ${ }^{15}$, recorrência da deformidade, pseudoartrose, fratura por osteoporose pós-imobilização ${ }^{18,36}$, perfuração pleural $^{24}$, fístula liquórica e anafilaxia ao látex ${ }^{29}$.

Niall et al. ${ }^{15}$ relatam complicações em 20 dos 24 pacientes tratados com cifosectomia e artrodese, com tempo médio de internação de 48 dias e perda sanguínea equivalente a $115 \%$ do volume total estimado do paciente durante o procedimento. Outros autores relatam perda sanguínea de 84 a $111 \%{ }^{24}$ e de $85 \%(875 \mathrm{~mL})^{35}$ da volemia. A magnitude do procedimento é confirmada pelos dados de Akbar et al. ${ }^{4}$, os quais relatam que 100\% dos pacientes necessitaram transfusões sanguíneas com perda estimada de $1.240 \mathrm{~mL}$, em média, durante o procedimento, com tempo médio de permanência de quatro dias na UTI após o procedimento, e por Martin et al. ${ }^{18}$ com perda sanguínea de $1.005 \mathrm{~mL}$ e duração cirúrgica de quatro horas e dez minutos, em média. Em recém-nascidos, a perda sanguínea foi de 118 a $253 \mathrm{~mL}^{12,14}$. Nos 11 recémnascidos tratados por Crawford et al. ${ }^{14}$, a duração média do procedimento foi de cinco horas e sete minutos (ortopedia e neurocirurgia no mesmo ato anestésico) com 27,2 dias de internação.

A taxa de infecção nesses pacientes costuma variar entre 33 a $45 \%{ }^{37}$. A perda de correção no pós-operatório pode ser progressiva, conforme relato de outros estudos ${ }^{35}$ : $17^{\circ} \mathrm{em}$ cinco anos e oito meses de seguimento e de $21,1^{\circ}$ aos 11 anos e 1 mês. No entanto, apesar do alto índice de complicações descrito, os resultados clínicos têm sido satisfatórios com obtenção do equilíbrio de tronco em 100\% das crianças com desaparecimento das úlceras ${ }^{4}$ e consolidação em todos os casos tratados pela técnica de DunnMcCarthy modificada associada a parafusos pediculares ${ }^{37}$ e nas revisões de pseudoartrose ${ }^{18}$. Crawford et al. ${ }^{14}$ creditam esses bons resultados clínicos à manutenção de cifose abaixo de $60^{\circ}$ ao final do crescimento e a uma modificação do padrão da cifose de uma deformidade de raio curto para uma cifose mais harmônica, de raio longo.
O crescimento longitudinal da coluna torácica entre os cinco e nove anos de idade é estimado em $0,6 \mathrm{~cm} / \mathrm{ano}$ numa criança normal ${ }^{38}$ e cerca de $50 \%$ do volume torácico final é obtido com o crescimento entre os 10 e 15 anos de idade $^{39}$. Assim, as técnicas tradicionais de tratamento em crianças de pouca idade e baixa estatura acarretam limitação do desenvolvimento tridimensional da caixa torácica ${ }^{40}$ e perda da altura do tronco com compressão do conteúdo abdominal contra o diafragma e, consequente, redução da capacidade pulmonar ${ }^{21}$. No entanto, a demora no tratamento leva a perda de independência funcional tanto pelo atraso no sentar e pela impossibilidade da utilização das mãos nessa posição sentada, já que os membros superiores estão sendo utilizados para sustentação do tronco pela falta de equilíbrio ${ }^{21}$.

A prótese VEPTR é um dispositivo que permite alongamentos periódicos. Foi desenvolvido para o tratamento da SIT nos pacientes esqueleticamente imaturos ${ }^{39,41}$ e permite a correção e estabilização indireta da deformidade, fixando-se na costela e na pelve. Seu uso tem se expandido para outras deformidades, sendo utilizado como um sistema de crescimento em escolioses congênitas ${ }^{41} \mathrm{e}$ em pacientes com escoliose neuromuscular ${ }^{42,43}$. Este não é um procedimento isento de complicações. Vitale et al. ${ }^{44}$ tiveram uma taxa de 30\% de complicações pós-operatórias com esse sistema, sendo infecção a mais frequente $(13,5 \%)$, seguida de soltura do implante $(8,1 \%)$, associação entre infecção e soltura $(5,4 \%)$ e complicações dermatológicas $(2,7 \%)$ em pacientes com fusão de costelas, hipoplasia torácica ou deformidades progressivas. Nos portadores de deformidades neuromusculares, a taxa relatada de complicação é de $23,5 \%{ }^{43}$.

O uso do VEPTR no tratamento da cifose congênita nos pacientes com mielomeningocele tem como objetivo corrigir a cifose e o balanço sagital, mantendo o controle dessa correção durante o crescimento. Trata-se de um procedimento cirúrgico que não acarreta manipulação da pele cicatricial do fechamento da bolsa, atrófica e sem sensibilidade na linha mediana e permite crescimento em altura do tronco, por não envolver ressecção de vértebras ou artrodese em crianças esqueleticamente imaturas. A manutenção de todas as vértebras associada à correção da cifose provoca um aumento do volume abdominal, permitindo um maior ganho de peso. $\mathrm{O}$ desenvolvimento das habilidades da criança é possível pela liberação dos membros superiores na posição sentada. Além da melhora do valor angular da deformidade, o uso do VEPTR proporcionou uma melhora no equilíbrio do tronco, tanto pela melhora no balanço sagital, como pela diminuição da anteriorização do tronco e pela diminuição da anteversão da pelve. O sangramento durante o procedimento também foi pequeno (não houve necessidade de transfusão sanguínea intra ou pós-operatória) e nenhum paciente permaneceu na UTI por mais de 24 horas no pós-operatório. Outra vantagem observada é o fato de os pontos de apoio do implante estarem localizados fora dos 
elementos ósseos da coluna vertebral, já que esses são em geral hipoplásicos. Essa hipoplasia gera dificuldades para uma fixação segura com manutenção da correção e, além disso, a simples manipulação desses elementos vertebrais pode provocar uma artrodese no local abordado, mesmo na instalação de sistemas de crescimento nessas crianças de baixa idade.

Sua indicação deve ser feita em concordância plena com os cuidadores da criança, já que essa técnica implica em internação e todos os riscos de um procedimento cirúrgico a cada quatro a seis meses para realização dos alongamentos, apesar de a taxa de complicações não ser maior do que a descrita para os outros procedimentos para correção da cifose congênita em crianças com mielomeningocele ${ }^{8,10,12,14,16,24-31}$. Com relação às complicações descritas para o VEPTR, não foi observado nenhum acometimento do plexo braquial, por evitar o apoio do sistema na primeira costela durante as montagens ou reposicionamento.

Utilizado como um sistema de crescimento espera-se que a criança atinja a maturidade esquelética em melhores condições clínicas e nutricionais pelo aumento do volume abdominal, com uma cifose de menor grau e mais flexível e com bom equilíbrio do tronco pela correção do balanço sagital. Tem sido observada a manutenção dos valores obtidos no pós-operatório ao longo do acompanhamento e dos alongamentos, com perda mínima da correção próxima à época do alongamento. Esse seguimento irá revelar a necessidade, ou não, da realização da cifosectomia e artrodese, tratamento definitivo para centralização da coluna sobre a bacia. Neste estudo, nenhuma criança atingiu ainda a maturidade esquelética para ser submetida a tal procedimento. Deverá ser observada, inclusive, a quantidade de vértebras a serem ressecadas para correção final da deformidade em comparação com as outras técnicas de vertebrectomia. Assim, será possível avaliar se a manutenção da cifose com um valor angular mais baixo representará menor morbidade ao procedimento.

\section{CONCLUSÃO}

A utilização do VEPTR nos pacientes portadores de mielomeningocele torácica com cifose congênita permite correção da cifose e do equilíbrio sagital em idade mais precoce, com manutenção dessa correção ao longo do seguimento, permitindo com isso desenvolvimento das habilidades manuais pela liberação dos membros superiores ao sentar, ganho de peso pelo aumento do volume abdominal e desaparecimento das úlceras de pressão.

O seguimento dos casos permitirá uma comparação com as técnicas tradicionais, mas parece tratar-se de uma técnica bastante promissora para tratamento mais precoce dessa deformidade extremamente limitante no desenvolvimento das crianças com mielomeningocele.

\section{REFERÊNCIAS}

1. Hoppenfeld S. Congenital kyphosis in myelomeningocele. J Bone Joint Surg Br. 1967;49(2):276-80.

2. Christofersen MR, Brooks AL. Excision and wire fixation of rigid myelomeningocele kyphosis. J Pediatr Orthop. 1985;5(6):691-6.

3. Banta JV. The evolution of surgical treatment of spinal deformity in myelomeningocele. Z Kinderchir. 1987;42 Suppl 1:10-2.

4. Akbar M, Bremer R, Thomsen M, Carstens C, Abel R. Kyphectomy in children with myelodysplasia: results 1994-2004. Spine (Phila Pa 1976). 2006;31(9):1007-13.

5. Raycroft JF, Curtis BH. Spinal curvature in myelomeningocele: natural historyand etiology. In: American Academy of Orthopedic Surgeons Symposium on myelomeningocele. St. Louis: C.V. Mosby; 1972.

6. Burney DW Jr, Hamsa WR. Spina Bifida with myelomeningocele. Clin Orthop Relat Res. 1963;30:167-74.
7. Naik DR, Lendon RG, Barson AJ. A radiological study of vertebral and rib malformations in children with myelomeningocele. Clin Radiol. 1978;29(4):427-30.

8. Carstens C, Koch H, Brocai DR, Niethard FU. Development of pathological lumbar kyphosis in myelomeningocele. J Bone Joint Surg Br. 1996;78(6):945-50.

9. Banta JV. Combined anterior and posterior fusion for spinal deformity in myelomeningocele. Spine (Phila $\mathrm{Pa}$ 1976). 1990;15(9):946-52.

10.Cotta H, Parsch K, Schulitz KP. [The treatment of lumbar kyphosis in spina bifida cystica]. Z Orthop Ihre Grenzgeb. 1971;108(4):567-74. German.

11.Banta JV, Hamada JS. Natural history of the kyphotic deformity in myelomeningocele. J Bone Joint Surg Am. 1976;58A:279.

12.Sharrard WJ. Spinal osteotomy for congenital kyphosis in myelomeningocele. J Bone Joint Surg Br. 1968;50(3):466-71.
13.Drennan JC. The role of muscles in the development of human lumbar kyphosis. Dev Med Child Neurol Suppl. 1970;22:Suppl 22:33-8.

14.Crawford AH, Strub WM, Lewis R, Gabriel KR, Bilmire DA, Berger T, et al. Neonatal kyphectomy in the patient with myelomeningocele. Spine (Phila Pa 1976). 2003;28(3):260-6.

15.Niall DM, Dowling FE, Fogarty EE, Moore DP, Goldberg C. Kyphectomy in children with myelomeningocele: a long-term outcome study. J Pediatr Orthop. 2004;24(1):37-44.

16.Heydemann JS, Gillespie R. Management of myelomeningocele kyphosis in the older child by kyphectomy and segmental spinal instrumentation. Spine (Phila $\mathrm{Pa}$ 1976). 1987;12(1):37-41.

17.Mazur J, Menelaus MB, Dickens DR, Doig WG. Efficacy of surgical management for scoliosis in myelomeningocele: correction of deformity and alteration of functional status. J Pediatr Orthop. 1986;6(5):568-75. 
18.Martin J Jr, Kumar SJ, Guille JT, Ger D, Gibbs M. Congenital kyphosis in myelomeningocele: results following operative and nonoperative treatment. J Pediatr Orthop. 1994;14(3):323-8.

19.Torre-Healy A, Samdani AF. Newer technologies for the treatment of scoliosis in the growing spine. Neurosurg Clin N Am. 2007;18(4):697-705.

20.Thompson GH, Akbarnia BA, Campbell RM Jr. Growing rod techniques in early-onset scoliosis. J Pediatr Orthop. 2007;27(3):354-61.

21.Sarwark JF. Kyphosis deformity in myelomeningocele. Orthop Clin North Am. 1999;30(3):451-5,viii-ix.

22.Mintz LJ, Sarwark JF, Dias LS, Schafer MF. The natural history of congenital kyphosis in myelomeningocele. A review of 51 children. Spine (Phila Pa 1976). 1991;16(8 Suppl):S348-50.

23.Sharrard WJ, Drennan JC. Osteotomyexcision of the spine for lumbar kyphosis in older children with myelomeningocele. J Bone Joint Surg Br. 1972;54(1):50-60.

24.Lindseth RE, Stelzer L Jr. Vertebral excision for kyphosis in children with myelomeningocele. J Bone Joint Surg Am. 1979;61(5):699-704.

25.McCarthy RE, Dunn H, McCullough FL. Luque fixation to the sacral ala using the Dunn-McCarthy modification. Spine (Phila Pa 1976). 1989;14(3):281-3.

26.McMaster MJ. The long-term results of kyphectomy and spinal stabilization in children with myelomeningocele. Spine (Phila Pa 1976). 1988;13(4):417-24.

27.Lowe GP, Menelaus MB. The surgical management of kyphosis in older children with myelomeningocele. J Bone Joint Surg Br. 1978;60(1):40-5.

28. Warner WC Jr, Fackler CD. Comparison of two instrumentation techniques in treatment of lumbar kyphosis in myelodysplasia. J Pediatr Orthop. 1993;13(6):704-8.

29.Nolden MT, Sarwark JF, Vora A, Grayhack JJ. A kyphectomy technique with reduced perioperative morbidity for myelomeningocele kyphosis. Spine (Phila Pa 1976). 2002;27(16):1807-13.
30. Sriram K, Bobechko WP, Hall JE. Surgical management of spinal deformities in spina bifida. J Bone Joint Surg Br. 1972;54(4):666-76.

31.Duncan JW, Lovell WW, Bailey SC, Ransom D. Surgical treatment of kyphosis in myelomeningocele. J Bone Joint Surg Am. 1976;58:155.

32.Leatherman KD, Dickson

RA. Congenital kyphosis in myelomeningocele. Vertebral body resection and posterior spine fusion. Spine (Phila Pa 1976). 1978;3(3): 222-6.

33.Ferraretto I, Mota DP, Fernandes AC, Machado PO, Silva Jr JA. Cifoses na mielomeningocele: conceitos, técnicas de tratamento e apresentação de casos. Rev Bras Ortop. 1998;33(12):963-72.

34.Hall JE, Poitras B. The management of kyphosis in patients with myelomeningocele. Clin Orthop Relat Res. 1977;(128):33-40.

35.Lintner SA, Lindseth RE. Kyphotic deformity in patients who have myelomeningocele. Operative treatment and long-term followup. J Bone Joint Surg Am. 1994;76(9):1301-7.

36.Doers T, Walker JL, van den Brink K, Stevens DB, Heavilon $\mathrm{J}$. The progression of untreated lumbar kyphosis and the compensatory thoracic lordosis in myelomeningocele. Dev Med Child Neurol. 1997;39(5):326-30.

37.Dias RCC, Veiga IG, Pasqualini W, Santos MAM, Landim E, Cavali PTM. Avaliação do tratamento cirúrgico da cifose congênita na mielomeningocele com o uso da via posterior pela técnica de Dunn-McCarthy modificada. Coluna/Columna. 2008;7(2):146-52.

38.Campbell RM Jr, Hell-Vocke AK. Growth of the thoracic spine in congenital scoliosis after expansion thoracoplasty. J Bone Joint Surg Am. 2003;85-A(3):409-20.

39. Campbell RM Jr, Smith MD, Mangos JA, et al. The treatment of thoracic insufficiency syndrome associated with progressive early onset scoliosis by opening wedge thoracostomy. Presented at: the Annual Meeting of the Scoliosis Research Society; Oct. 27-30; Miami, FL, 2005.
40.Emans JB, Caubet JF, Ordonez CL, Lee EY, Ciarlo M. The treatment of spine and chest wall deformities with fused ribs by expansion thoracostomy and insertion of vertical expandable prosthetic titanium rib: growth of thoracic spine and improvement of lung volumes. Spine (Phila Pa 1976). 2005;30(17 Suppl):S58-68.

41.Campbell RM Jr, Smith MD, Mayes TC, Mangos JA, Willey-Courand DB, Kose N, et al. The effect of opening wedge thoracostomy on thoracic insufficiency syndrome associated with fused ribs and congenital scoliosis. J Bone Joint Surg Am. 2004;86-A(8):1659-74.

42.Hell AK, Campbell RM, Hefti F. The vertical expandable prosthetic titanium rib implant for the treatment of thoracic insufficiency syndrome associated with congenital and neuromuscular scoliosis in young children. J Pediatr Orthop B. 2005; 14(4):287-93.

43.Landim E, Cavali PTM, Santos MAM, Pasqualini W, Boechat RCBM, Andrade SMS. Uso da prótese vertical expansível de titânio para costela (VEPTR) com opção na instrumentação sem fusão para tratamento da escoliose neuromuscular. Coluna/Columna. 2008;7(2):160-6.

44. Vitale MG, Matsumoto H, Roye DP Jr, Gomez JA, Betz RR, Emans JB, et al Health-related quality of life in children with thoracic insufficiency syndrome. J Pediatr Orthop. 2008;28(2):239-43.

\section{Correspondência}

Guilherme Rebechi Zuiani

Avenida Andrade Neves, 699, $2^{\circ}$ andar, conjunto 22

CEP: 13013-161 - Campinas (SP), Brasil

Tel: (19) 3236-4485

Email:grzuiani@superig.com.br 